\title{
BMJ Open MASked-unconTrolled hypERtension management based on office BP or on ambulatory blood pressure measurement (MASTER) Study: a randomised controlled trial protocol
}

To cite: Parati G, AgabitiRosei E, Bakris GL, et al. MASked-unconTrolled hypERtension management based on office BP or on ambulatory blood pressure measurement (MASTER) Study: a randomised controlled trial protocol. BMJ Open 2018;8:e021038. doi:10.1136/ bmjopen-2017-021038

- Prepublication history and additional material for this paper are available online. To view these files, please visit the journal online (http://dx.doi. org/10.1136/bmjopen-2018021038).

AZ died on 24 March 2018.

Received 21 December 2017 Revised 6 June 2018 Accepted 25 0ctober 2018

Check for updates

(c) Author(s) (or their employer(s)) 2018. Re-use permitted under CC BY-NC. No commercial re-use. See rights and permissions. Published by BMJ.

For numbered affiliations see end of article.

Correspondence to Dr Gianfranco Parati; gianfranco.parati@unimib.it

Gianfranco Parati, ${ }^{1,2,3}$ Enrico Agabiti-Rosei, ${ }^{4,5}$ George L Bakris, ${ }^{6,7}$ Grzegorz Bilo, ${ }^{2}$ Giovanna Branzi, ${ }^{2}$ Franco Cecchi, ${ }^{8}$ Marzena Chrostowska, ${ }^{9}$ Alejandro De la Sierra, ${ }^{10}$ Monica Domenech, ${ }^{11}$ Maria Dorobantu, ${ }^{12}$ Thays Faria, ${ }^{2}$ Yong Huo, ${ }^{13}$ Bojan Jelaković, ${ }^{14}$ Thomas Kahan, ${ }^{15,16}$ Alexandra Konradi, ${ }^{17}$ Stéphane Laurent, ${ }^{18}$ Nanfang Li, ${ }^{19}$ Kushal Madan, ${ }^{20}$ Giuseppe Mancia, ${ }^{1}$ Richard J McManus, ${ }^{21}$ Pietro Amedeo Modesti, ${ }^{22}$ Juan Eugenio Ochoa, ${ }^{2}$ José Andrés Octavio, ${ }^{23}$ Stefano Omboni, ${ }^{24}$ Paolo Palatini, ${ }^{25}$ Jeong Bae Park, ${ }^{26}$ Dario Pellegrini, ${ }^{1}$ Sabine Perl, ${ }^{27}$ Cristian Podoleanu, ${ }^{28}$ Giacomo Pucci, ${ }^{29,30}$ Josep Redon, ${ }^{31,32}$ Nicolas Renna, ${ }^{33}$ Moo Yong Rhee, ${ }^{34}$ Enrique Rodilla Sala, ${ }^{35}$ Ramiro Sanchez, ${ }^{36}$ Roland Schmieder, ${ }^{37}$ Davide Soranna, ${ }^{2,38}$ George Stergiou, ${ }^{39,40}$ Milos Stojanovic, ${ }^{41}$ Konstantinos Tsioufis, ${ }^{42}$ Maria Grazia Valsecchi, ${ }^{43}$ Franco Veglio, ${ }^{44}$ Gabriel Dario Waisman, ${ }^{45} \mathrm{Ji}$ Guang Wang, ${ }^{46,47}$ Paulina Wijnmaalen, ${ }^{2}$ Antonella Zambon, ${ }^{2,38}$ Alberto Zanchetti, ${ }^{2}$ Yuqing Zhang ${ }^{48}$

\section{ABSTRACT}

Introduction Masked uncontrolled hypertension (MUCH) carries an increased risk of cardiovascular (CV) complications and can be identified through combined use of office $(0)$ and ambulatory (A) blood pressure (BP) monitoring (M) in treated patients. However, it is still debated whether the information carried by ABPM should be considered for MUCH management. Aim of the MASked-unconTrolled hypERtension management based on $\mathrm{OBP}$ or on ambulatory blood pressure measurement (MASTER) Study is to assess the impact on outcome of MUCH management based on OBPM or ABPM. Methods and analysis MASTER is a 4-year prospective, randomised, open-label, blinded-endpoint investigation. A total of 1240 treated hypertensive patients from about 40 secondary care clinical centres worldwide will be included -upon confirming presence of MUCH (repeated on treatment $\mathrm{OBP}<140 / 90 \mathrm{~mm} \mathrm{Hg}$, and at least one of the following: daytime $A B P \geq 135 / 85 \mathrm{~mm} \mathrm{Hg}$; night-time $A B P \geq 120 / 70 \mathrm{~mm}$ $\mathrm{Hg}$; 24 hour $\mathrm{ABP} \geq 130 / 80 \mathrm{~mm} \mathrm{Hg}$ ), and will be randomised to a management strategy based on OBPM (group 1) or on ABPM (group 2). Patients in group 1 will have OBP measured at $0,3,6,12,18,24,30,36,42$ and 48 months and taken as a guide for treatment; $A B P M$ will be performed at randomisation and at 12, 24,36 and 48 months but will not be used to take treatment decisions. Patients randomised to group 2 will have ABPM performed at randomisation and all scheduled visits as a guide to antihypertensive treatment. The effects of MUCH management strategy based on ABPM or on OBPM on CV and renal intermediate outcomes (changing left ventricular mass and microalbuminuria, coprimary outcomes) at 1 year and on

\section{Strengths and limitations of this study}

- The MASked-unconTrolled hypERtension management based on office BP (OBP) or on ambulatory blood pressure measurement Study is the first randomised controlled study so far addressing an important and still controversial issue, that is, whether using ambulatory $\mathrm{BP}$ monitoring rather than $\mathrm{OBP}$ measurements as a guide to antihypertensive treatment confers any benefit in terms of cardiovascular prevention.

- For practical reasons, a double-blind study design could not be implemented.

- Due to the multicentre nature of our study which includes hypertension centres from all over the world, there is the theoretical possibility of a dropout rate larger than expected. For this reason, however, we have implemented a strict study monitoring web-based.

CV events at 4 years and on changes in BP-related variables will be assessed.

Ethics and dissemination MASTER study protocol has received approval by the ethical review board of Istituto Auxologico Italiano. The procedures set out in this protocol are in accordance with principles of Declaration of Helsinki and Good Clinical Practice guidelines. Results will be published in accordance with the CONSORT statement in a peer-reviewed scientific journal.

Trial registration number NCT02804074; Pre-results. 


\section{INTRODUCTION}

The finding in untreated hypertensives of normal blood pressure (BP) levels when measured in the medical office accompanied by elevated out-of-office BP as assessed by either 24hour ambulatory BP monitoring (ABPM) or home BP monitoring (HBPM) is defined as 'masked hypertension'. This condition is relatively common, characterising $10 \%-15 \%$ of individuals in a population. ${ }^{1}$ Remarkably, even after initiation of antihypertensive treatment, a substantial number of patients continue to show elevated BP levels out of the office, despite having apparently well-controlled BP levels during the medical visit. This condition has been defined as 'masked uncontrolled hypertension' (MUCH), and in a population of hypertensive patients under treatment has been reported to occur in as much as $30 \%-31 \%$ of cases. ${ }^{2}$ Moreover, there is evidence that prescription of antihypertensive treatment without adequate care of 24-hour BP coverage and, without implementation of ABPM in the assessment of treatment effects, may indeed be responsible for an increase in the frequency of $\mathrm{MUCH}^{3}$ As in the case of $\mathrm{MH}, \mathrm{MUCH}$ has been associated with an increased cardiovascular (CV) risk which is very similar to that of sustained hypertension and sustained uncontrolled hypertension in treated patients (ie, persistent elevation in both office and out-of-office BP levels). ${ }^{34}$ Several hypertension guidelines have indeed included suspicion of these conditions among the key clinical indications for out-of-office BP monitoring. ${ }^{5-11}$ The superiority of out-of-office $\mathrm{BP}$ versus office $\mathrm{BP}$ (OBP) measurements has been recently emphasised also by hypertension management guidelines, in particular by the National Institute of Health and Care Excellence guidelines which have recommended use of a time-restricted ABPM before starting treatment in patients with elevated OBP. ${ }^{12}$ However, key evidence from randomised ad hoc intervention trials on the benefits of using out-of-office $\mathrm{BP}$ measurement for assessment of BP control during the follow-up of treated hypertensive patients is still lacking. With the aim to better explore this issue, the MASTER Study (MASked-unconTrolled hypERtension management based on office BP or on ambulatory blood pressure measurement) will evaluate whether an ABPMbased hypertension management strategy is superior to an OBP monitoring (OBPM)-based strategy in changing left ventricular mass (LVM) and microalbuminuria (coprimary outcomes) at 1 year, in preventing CV events (secondary outcome) at 4 years and in improving several BP-related variables throughout the study. The MASTER study, focusing on MUCH patients, is thus expected to provide useful information aimed at finally clarifying whether a management strategy based on out-of-office BP measurements might provide a greater benefit in terms of prevention or regression of organ damage and CV events than a management strategy based on OBP readings only, thereby assessing the actual value of using out-of-office BP in improving CV protection.

\section{METHODS}

\section{Study design}

The present study is a 4-year prospective, randomised, open-label, blinded-endpoint (PROBE) study aimed at comparing a management strategy for patients with MUCH, based on OBPM as a guide to antihypertensive treatment (group 1) versus a management strategy based on use of ABPM for the same purpose (group 2). The study will have a period of 2 years for the enrolment of the requested number of patients; and an average 4-year follow-up period ( 3 to 5 years).

Study endpoints are changes in LVM and microalbuminuria (coprimary outcomes) at 1 year, prevention of CV events including all-cause mortality, CV morbidity and mortality (secondary outcomes) at 4 years and improvement of several BP-related variables throughout the study (tertiary outcome). Following a parallel group study design, patients will be randomised to one of the two management strategies by a centralised computer-generated sequence with an allocation ratio of $1: 1$.

\section{Sample selection}

\section{Recruitment}

MASTER is a multicentre, multinational study including around 40 clinical centres from different continents: Europe (Austria, Belgium, Croatia, Georgia, Germany, Italy, Poland, Romania, Russia, Serbia, Spain, Sweden), Asia (China, India, Korea) and South America (Argentina, Brazil, Venezuela). A list with the participating centres is provided in the online supplementary file 1.

To all participating centres, the coordinating centre in Milan will supply the due study documentation, including a description of the protocol and of the expected tasks, and the files to be signed in relation to study participation agreement. In order to guarantee the necessary data quality, each participating centre will be asked to submit to the coordinating centre information on the available devices for collection of the data required by the protocol. They will also be asked to send a sample of the recorded files related to specific study variables for quality check and file approval by the coordinating centre team. This will be specifically required for digital ECG files, for cardiac ultrasound records, for 24 hour ABPM files and for $\mathrm{HBP}$ records. If the centre fulfils all requirements and complies with study quality standards, an official agreement will be signed with the coordinating centre before trial can be started. Approval by local ethics committee will also be required for each participating centre.

\section{Screening and randomisation}

Around 40 subjects will be enrolled in each centre upon verifying the study eligibility criteria listed in box 1 .

Patients are to be enrolled for screening and randomisation over 1 year (a maximum of 2 years will be allowed). During the screening period ( 1 month before randomisation), eligibility of the patient will be checked and baseline measurements performed. Initial assessment of selection criteria based on subjects' clinical history will take place 
Box 1 Inclusion and exclusion criteria for enrolment and exclusion criteria for enrolment

\section{Inclusion criteria \\ - Male and female subjects; \\ - Age 40-80 years; \\ - Diagnosis of masked uncontrolled (on treatment) hypertension: of- fice blood pressure (BP) $<140 / 90 \mathrm{~mm} \mathrm{Hg}$, and one or more of the following situations: \\ - Ambulatory daytime BP $\geq 135 / 85 \mathrm{~mm} \mathrm{Hg}$ \\ - Ambulatory night-time BP $\geq 120 / 70 \mathrm{~mm} \mathrm{Hg}$ \\ - Ambulatory 24 hours BP $\geq 130 / 80 \mathrm{~mm} \mathrm{Hg}$ \\ - Estimated glomerular filtration rate (eGFR) $\geq 45 \mathrm{~mL} / \mathrm{min} / 1.73 \mathrm{~m}^{2}$ (Chronic Kidney Disease Epidemiology Collaboration (CKD-EPI) cre- atinine equation 2009).}

\section{Exclusion criteria}

- eGFR $<45 \mathrm{~mL} / \mathrm{min} / 1.73 \mathrm{~m}^{2}$ (CKD-EPI creatinine equation 2009), and in particular severe chronic renal failure defined as serum creatinine $>250 \mu \mathrm{mol} / \mathrm{L}$;

- One of the following conditions:

- Persistent atrial fibrillation

- Evidence of severe cardiac valve disease (ie, $>2 / 4$ grade at echocardiographic examination)

- Moderate and severe aortic stenosis

- Presence of cardiomyopathy

- Symptomatic heart failure or ejection fraction at or below $45 \%$

- Patients in unstable clinical conditions;

- Known secondary hypertension;

- Orthostatic hypotension (systolic blood pressure fall $>20 \mathrm{~mm} \mathrm{Hg}$ on standing);

- Dementia (clinical diagnosis);

- Hepatic disease as determined by either aspartate transaminase or alanine transaminase values $>2$ times the upper reference limit;

- History of gastrointestinal surgery or disorders which could interfere with drug absorption;

- Known allergy or contraindications to one of the drugs to be administered in the study;

- History of malignancy including leukaemia and lymphoma (but not basal cell skin cancer) within the last 5 years;

- History of clinically significant autoimmune disorders such as systemic lupus erythematosus;

- History of drug or alcohol abuse within the last 5 years;

- History of non-compliance to medical regimens and/or patients who are considered potentially unreliable;

Inability or unwillingness to give free informed consent

Pregnancy or planned pregnancy during study period.

and, in case the subject is potentially eligible, the participation in the study will be proposed and informed consent obtained. During one or more screening visits, according to the need of the enrolling unit, the additional evaluations needed for a complete assessment of eligibility criteria will be performed, ie, full medical history, physical examination, blood and urine samples, conventional BP measurements, ABPM placement for 24 hour recording (device to be removed on the following day). Once the diagnosis of MUCH has been made, baseline study variables will be collected. These include 7 days HBPM through use of oscillometric devices validated by means of international protocols, ECG and echocardiographic examinations and blood and urine tests. Blood samples will be obtained for assessment of estimated glomerular filtration rate (eGFR), plasma creatinine, plasma glucose, glycated haemoglobin (HbAlc), uric acid, serum sodium and potassium and plasma lipids. Two blood samples will also be collected at follow-up visit 1 and at follow-up visit 3 in order to create a study biobank for future analyses. Spot urine samples for urine albumin:creatinine ratio (UACR) will be taken twice, once in the morning before application of the ABPM device, and another time on the following morning when subjects will return to the clinical center for removing the ABPM device.

At each study site, the responsible investigator will arrange a randomisation visit (within 1 month after the screening period) during which eligible patients (ie, those found to have $\mathrm{MUCH}$ ) will be randomised to one of the two study groups following the dynamic allocation method for balancing baseline covariates (specifically centre, age, sex, presence of diabetes and baseline office systolic BP (SBP)) proposed by Xhiao et al in 2012. ${ }^{13}$ The patient's randomisation number and allocation will be generated by a central computer using an online algorithm inbuilt on the electronic-Clinical Report Form (e-CRF). This approach adapts the algorithm proposed by Frane $e t$ al to obtain marginal balance for both continuous and categorical covariates, adding Efron's biased coin method to decrease the predictability of treatment assigned to a new patient. ${ }^{14}$ This aspect is very important especially for unblinded trials. The original $\mathrm{R}$ code to perform this algorithm, furnished by the author, will be set on the platform managing the e-CRF.

\section{Blinding (masking)}

Because the MASTER is an open-label study, blinding will apply only for the endpoints. Thus, both participants and investigators will not be blinded to the allocated intervention. In order to guarantee the blind assessment of the coprimary end point of LVM, the personnel involved in its assessment (ie, sonographers) will be blinded to the patient's allocated management strategy. In addition, ABPM data of patients enrolled to group 1 will be directly uploaded on the e-CRF and will not be available to the investigators in charge of treatment decisions. The role of the Event Adjudicating Committee (EAC) of the MASTER Study is essential in a trial such as MASTER, necessarily designed as a PROBE trial. EAC within the MASTER study will be responsible for verifying and adjudicating in a blinded fashion all events representing primary, secondary and tertiary outcomes of the study, as well as all serious adverse events occurring in the course of the trial. For this reason, the General Coordinating Centre in Milan will insure that all outcome documentations sent to EAC is blinded for management strategy and intensity of antihypertensive therapy. Documentation of each outcome occurring in patients will be sent (electronically or by fax) to two experts and to a third one in case the first two do not reach an agreement. Only validated outcomes will enter the final data analysis. 


\section{Interventions}

Blood pressure levels in both study groups will be measured by three BP measurement techniques (OBP, HPB and 24 hour ABPM) following the recommendations issued by the European Society of Hypertension/European Society of Cardiology hypertension guidelines ${ }^{5}$ and BP monitoring groups. ${ }^{6-8}$ Indications on how to measure BP in the office, at home and in ambulatory conditions over 24 hours are provided in the supplemental operation manual. In order to standardise ABPM and OBPM data collection and analysis as much as possible, 24hours ABPM in MASTER Study will be preferentially performed with A\&D TM2430 device and OBP levels will be measured with A\&D UA-651BLE device provided by the coordinating centre, thanks to a non-conditioning support given by the A\&D Company (A\&D Engineering, San Jose, California, USA). For HBPM, any of the currently available validated devices can be employed. Patients randomised to be managed based on OBP (group 1) will have their BP measured by the office method at all scheduled visits (at 0, 3, 6, 12, 18, 24, 30, 36, 42 and 48 months). In these patients, ABPM will only be performed at randomisation visit and at follow-up visits 3, 5, 7 and 9 and in case of premature discontinuation (ie, patients' decision to withdraw, non-compliance with the study protocol) at any time. However, ABP values will not be used by the investigator to take treatment decisions, but treatment will be based on OBP only. According to the ABPM procedures manual of the MASTER Study, data of these patients will not be made available to the investigators in charge of treatment decisions, but will be directly uploaded on the study website (table 1). As indicated in table 1, additional tests and examinations will be performed at different time points during the study follow-up. For assessment of LV mass and function, both B mode and M mode two-dimensional (2D) echocardiographic measurements and calculation of LVM will be performed according to current American Society of Echocardiography guidelines. ${ }^{15-18}$ LVM will be calculated with the formula for estimation of LVM from LV linear dimensions. ${ }^{18}$ In order to account for obesity-related LV hypertrophy (LVH), ${ }^{16} 17$ correction of LVM will be performed both for height and for body surface area (BSA) using the Dubois and Dubois formula. ${ }^{15} 17$ Since LVM values differ between men and women, with the latter being systematically lower than the former, even when indexed for BSA, the presence of $\mathrm{LVH}$ will be defined on the basis of upper limits of normality for LVM of $>115 \mathrm{~g} / \mathrm{m}^{2}\left(>48 \mathrm{~g} / \mathrm{m}^{2.7}\right)$ in men and $>95 \mathrm{~g} / \mathrm{m}^{2}\left(>44 \mathrm{~g} / \mathrm{m}^{2.7}\right)$ in women following recommendations for cardiac chamber quantification by echocardiography in adults. ${ }^{18}$ Patterns of LVH (concentric vs eccentric) and remodelling will also be considered. LV systolic function will be estimated by calculating ejection fraction by means of the biplane method of disks summation (modified Simpson's rule), which is the recommended 2D echocardiographic method by consensus. ${ }^{18}$ Diastolic function will also be assessed by considering the following parameters: mitral inflow pulsed wave (PW) Doppler, Doppler Tissue Imaging (DTI) of mitral annulus, PW Doppler of pulmonary vein flow, tricuspidal valve velocity peak calculated by continuous wave (CW) Doppler and indexed left atrial volume (biplane maximal left atrial volume will be calculated using the arealength method, and then indexed by BSA) as indicated by current guidelines. 1920

ECG recordings will also be performed at different time points of the study in order to identify the presence of ECG LVH. This will be done by means of specific indices such as Cornell Voltage Product and Index and SokolowLyon Index.

The coprimary end point of urinary albumin excretion (UAE) will be evaluated from morning spot urine samples and expressed as UACR $(\mathrm{mg} / \mathrm{g})$. Spot urine samples will be taken twice, once in the morning before application of the ABPM device, and another time on the following morning when the ABPM device will be removed.

Blood samples will also be obtained for determination of serum creatinine, glucose, $\mathrm{HbA1c}$, uric acid, $\mathrm{Na}+$, $\mathrm{K}+$ and lipids as well as to create a study biobank.

At each follow-up visit, physicians will not only ascertain achievement of BP control and/or adjust antihypertensive treatment if needed, but they will also record the type of antihypertensive therapy, presence of concomitant medications and adverse events on the study e-CRF for study safety evaluation (Table 1).

Given that the trial inclusion criteria establish that all patients in MASTER should have OBP controlled by treatment (ie, systolic OBP $<140$ and diastolic OBP $<90 \mathrm{~mm} \mathrm{Hg}$ ), all patients in group 1 will have their antihypertensive treatment unmodified at the randomisation visit. If at any of the subsequent trial visits, SBP or DBP will exceed normal values (ie, $\mathrm{SBP} \geq 140$ or $\mathrm{DBP} \geq 90 \mathrm{~mm} \mathrm{Hg}$ ), antihypertensive treatment will be intensified according to available guidelines (figure 1). Patients randomised to be managed based on ABPM (group 2) will have their BP measured by the ambulatory method at all scheduled visits. OBP will also be measured at all visits, but it will not be used by the investigator to take treatment decisions. Given that the trial inclusion criteria establish that all patients in MASTER should have uncontrolled ambulatory SBP or diastolic BP (DBP) values (24hour or daytime or night-time), all patients in group 2 will have their antihypertensive treatment intensified at the randomisation visit and at any of the subsequent trial visits, in which ambulatory SBP or DBP will exceed normal values (ie, 24 hour SBP $\geq 130$ or 24 hour DBP $\geq 80 \mathrm{~mm} \mathrm{Hg}$; daytime SBP $\geq 135$ or daytime DBP $\geq 85$; night-time SBP $\geq 120$ or nighttime DBP $\geq 70 \mathrm{~mm} \mathrm{Hg}$ ), according to available guidelines (figure 1). In case only daytime or night-time target values are not achieved, change in timing of doses to morning or evening, respectively, should be considered. At each follow-up visit, achievement of $\mathrm{ABP}$ control will be ascertained and antihypertensive treatment will be adjusted if needed, according to guidelines (figure 1). Patients' adherence will also be encouraged and monitored.

\section{Sample size calculation}

Sample size calculation was based on the primary study endpoint of changes in left ventricular mass index (LVMI) at 12 th month. We do not know what proportion of the 


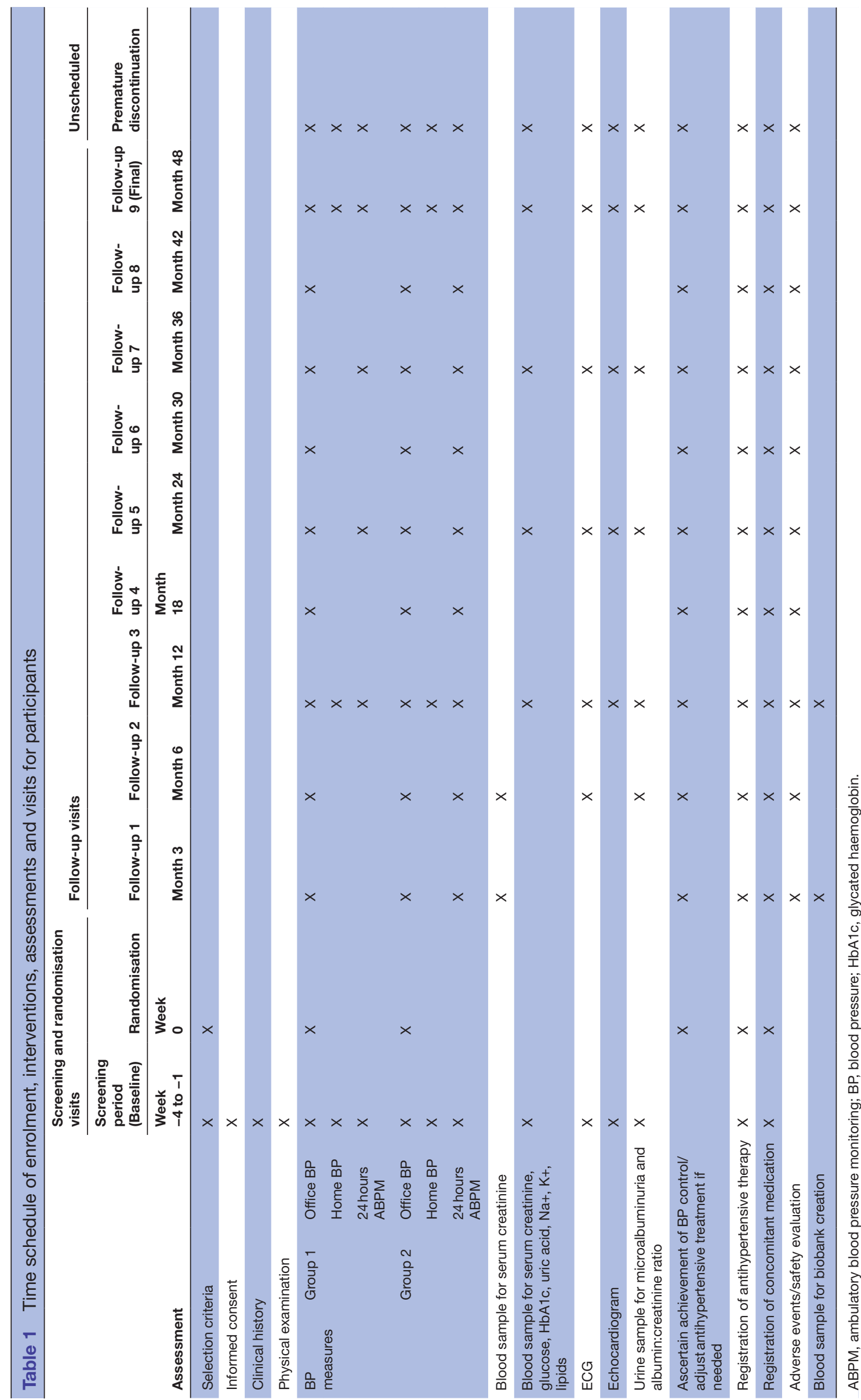

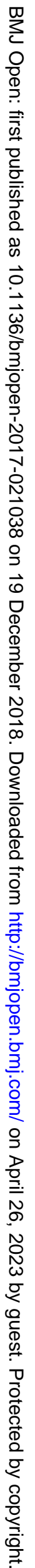




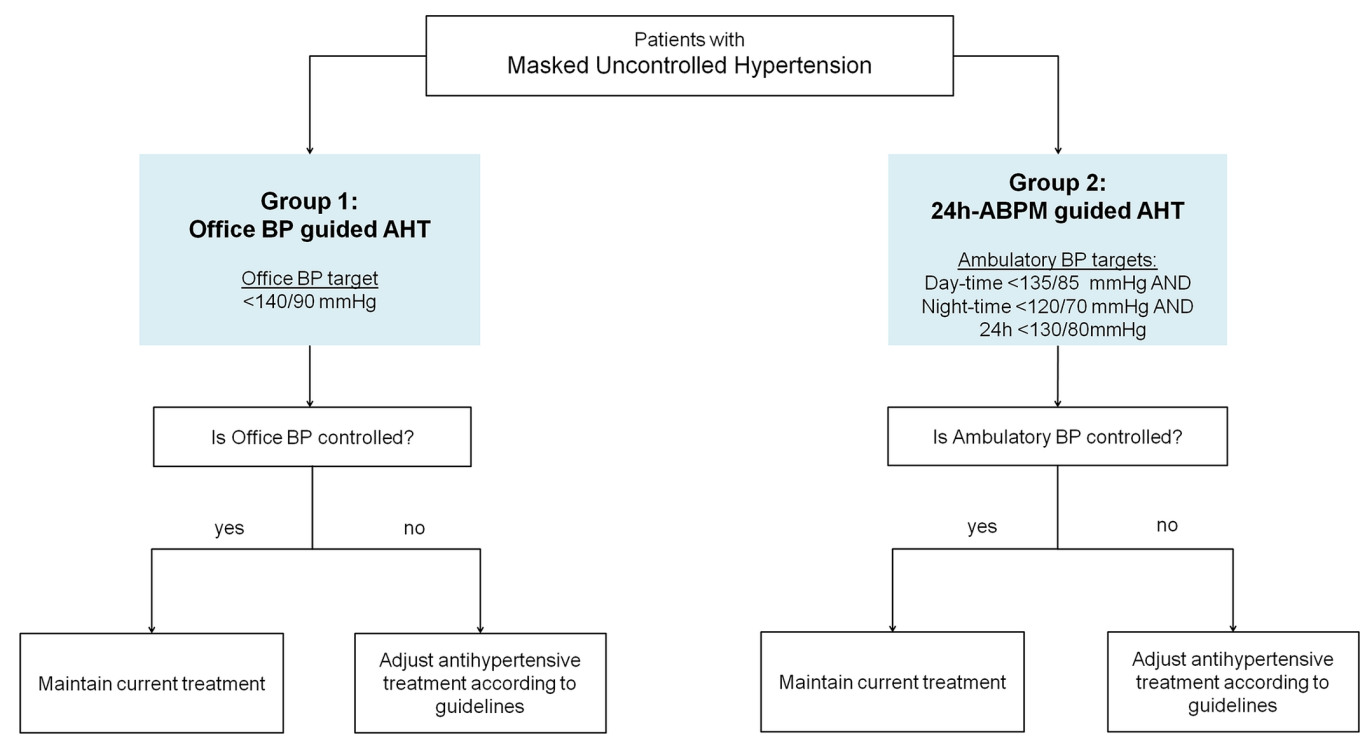

Figure 1 Randomisation groups and patient flow in the study. ABPM, ambulatory blood pressure monitoring; AHT, antihypertensive treatment; BP, blood pressure.

enrolled patients will have a diagnosis of LVH; therefore, the primary endpoint has not been based on categorical changes in LVH but rather on changes in LVM as a continuous variable. As the enrolled individuals are hypertensive patients with BP incompletely controlled by treatment, we expect LVM to be often higher than in subjects with sustained BP control (normal OPB and ABP levels). To calculate the sample size, we have assumed a difference of $5 \mathrm{~g} / \mathrm{m}^{2}$ in the change of LVMI with the ABP-guided (group 2) compared with the OBP-guided (group 1) management strategy. This has been based on the consideration that SBP will be reduced by about $8 \mathrm{~mm} \mathrm{Hg}$ more in the ABP-guided group, as well as on the echocardiographic data of studies such as REGAAL and CATCH in which differences in echo LVMI changes between treatments producing similar BP reductions were lower than $3 \mathrm{~g} /$ $\mathrm{m}^{2}{ }^{20}{ }^{21}$ The SD of $\pm 25 \mathrm{~g} / \mathrm{m}^{2}$ was also derived from data of REGAAL and CATCH as well as from previous assessment of within operator reproducibility. ${ }^{21} 22$ Considering a LVMI difference between groups of $5.0 \mathrm{~g} / \mathrm{m}^{2}$ and a SD of $25 \mathrm{~g} / \mathrm{m}^{2}$, an alpha of 0.025 (one-sided t-test) and a power (1-beta) of $90 \%$, with a dropout rate of $15 \%$ during the study period, a minimum of 620 subjects per study group was deemed necessary, for a total number of 1240 subjects to be randomised. However, given the uncertainty about the true LVMI difference we might observe and/or the related SD, performance of an interim analysis has been decided aimed at a possible re-estimation of the sample size. This analysis will be carried out at 18 th month when about $30 \%$ of randomised patients will achieve 1 year of follow-up. A re-estimation of the sample size will be calculated using the method of conditional power. ${ }^{23}$

\section{Outcomes}

Primary, secondary and tertiary outcomes are listed in box 2.

\section{Patient and public involvement}

Neither patients nor public have been involved during the design of MASTER Study. We only explored, in a pilot survey, how often OBP control was accompanied by persistence of elevated $\mathrm{BP}$ values at home in a number of patients referred to our hypertension centre. Results of MASTER study will be published in peer-reviewed scientific journals regardless of the outcome. Besides, MASTER study results will be available at https:/ / clinicaltrials.gov/ either to patients or general public. Finally, assessment of the burden of the intervention has not been foreseen in the present study.

\section{Data collection and data statement}

All data collected locally at each enrolling unit will be recorded into an electronic e-CRF on the website of the trial (https://master.digitalcrf.eu). Clinical data to be input into the e-CRF include demographic information, results on blood and urine laboratory tests performed locally (ie, plasma creatinine and eGFR, fasting glucose, uric acid, $\mathrm{Na}+, \mathrm{K}+$, Lipid profile, HbA1c, urinary sediment), ongoing antihypertensive treatment (number and dose of antihypertensive drugs), report forms for adverse events during follow-up. Digital files containing raw data from imaging (echocardiograms) and device-based examinations (ABPM, HBPM data, ECG tracings) will also be uploaded on the study website linked to the study e-CRF for further analysis by the central reading sites.

\section{Data management}

Clinical data and recordings obtained with the various technologies will be uploaded in the study e-CRF and on the study website under online control by the Data Management Coordinating centre, which includes an audit trail system. The quality of data obtained with the different technologies (ie, echocardiograms, ECG, ABPM, HBPM) will be validated by expert personnel at 


\section{Box 2 Study outcomes}

\section{Coprimary outcomes}

1. Changes from baseline to 12th month in echo left ventricular mass index (LVMI, $\mathrm{g} / \mathrm{m}^{2}$ ).

2. Changes from baseline to 12th month in urine albumin:creatinine ratio (UACR, mg/g, morning spot sample).

\section{Secondary outcomes}

1. As compared with baseline, presence or absence of echo left ventricular hypertrophy (LVH) (LVMI $\geq 115 \mathrm{~g} / \mathrm{m}^{2}$ (men) or $\geq 95 \mathrm{~g} / \mathrm{m}^{2}$ (women)) at 12th, 24th, 36th and 48th month.

2. Changes in ECG indices of LVH (Cornell Product (main index), Cornell Voltage Index, Sokolow-Lyon Score) from baseline to 12th, 24th, 36th and 48th month.

3. As compared with baseline, presence or absence of ECG LVH: (Sokolow-Lyon Index $\geq 3.5 \mathrm{mV}$ or Cornell Voltage Index $\geq 2.4 \mathrm{mV}$ (men) or $\geq 2.0 \mathrm{mV}$ (women) or Cornell Voltage Product $\geq 244 \mathrm{mVms}$ ) at 12th, 24th, 36th and 48th month.

4. Increase of microalbuminuria (defined as increase in UACR by $100 \%$ ) from baseline to 12 th, 24th, 36th and 48th month.

5. Decrease of microalbuminuria (defined as reduction in UACR by $50 \%$ ) from baseline to 12th, 24th, 36th and 48th month.

6. Changes in estimated glomerular filtration rate (eGFR) (mL/ $\mathrm{min} / 1.73 \mathrm{~m}^{2}$ ), according to Chronic Kidney Disease Epidemiology Collaboration (CKD-EPI) creatinine equation (2009) from baseline to 12th, 24th, 36th and 48th month.

7. As compared with baseline, presence or absence of CKD (eGFR $<60 \mathrm{~mL} / \mathrm{min} / 1.73 \mathrm{~m}^{2}$ ) at 12th, 24th, 36th and 48th month.

8. The first occurrence of a composite endpoint of events including fatal and non-fatal stroke, acute myocardial infarction, angina, revascularisation procedures (coronary, carotid, iliaco-femoral), transient ischaemic attack, atrial fibrillation, cardiovascular death, hospitalisation for heart failure, progression to severe CKD $\left(\right.$ GGFR $<30 \mathrm{~mL} / \mathrm{min} / 1.73 \mathrm{~m}^{2}$ (CKD-EPI creatinine equation (2009)), or doubling serum creatinine until 48th month.

9. Changes from baseline in echo LVMI at 24th, 36th and 48th month.

10. Changes from baseline in UACR at 24th, 36th and 48th month.

\section{Tertiary outcomes}

1. Changes from baseline in office blood pressure (OBP) at 3rd, 6th, 12th, 24th, 36th and 48th month.

2. Changes from baseline in ambulatory blood pressure (ABP; 24 hours, daytime and night-time BP) at 12th, 24th, 36th and 48th month.

3. Changes from baseline in home blood pressure (HBP) at 12th and 48th month.

4. Proportions of patients with controlled BP measured with OBP, HBP or ABP, respectively, at 12 th and 48th month.

5. Proportions of patients with controlled BP measured with OBP and $\mathrm{ABP}$ at 3,6,12 and 48 months (only for $\mathrm{ABP}$ group). Proportions of patients with controlled BP measured with $\mathrm{OBP}$ and HBP at 3, 12 and 48 months (for both groups).

6. Number of prescribed antihypertensive drugs at 3rd, 6th, 12th and 48th month.

7. Changes over time in the diagnosis of masked uncontrolled hypertension (on the basis of $\mathrm{ABPM}+\mathrm{OBP}$ ) at 12th and 48th month.

8. Comparison of the prevalence of masked uncontrolled hypertension (on the basis of $\mathrm{HBPM}+0 \mathrm{BP}$ or of $\mathrm{ABPM}+0 \mathrm{BP}$, respectively) at 12 th and at the 48th month.

9. Comparison between groups in visit-by-visit BP variability (standard deviation (SD); Coefficient of variation (CV); variability independent

Continued

\section{Box 2 Continued}

of the mean (VIM); average real variability (ARV)).

10. Comparison between groups in 24 hour BP variability ( 24 hours SD; 24 hours weighted SD (wSD); ARV) at 12th, 24th, 36th and 48th month.

11. Comparison between groups in day-by-day BP variability (SD, VC, ARV, maximun (MAX) BP) at 12th and 48th month.

12. Comparison between groups in Smoothness Index and treatment-on-variability index (TOVI) at 12th and 48th month.

13. Comparison between groups in the correlation between changes from baseline to 12th, 24th, 36th and 48th month in LVMI and changes from baseline to 12th, 24th, 36th and 48th month of different measures of $\mathrm{BP}(\mathrm{OBP}, \mathrm{ABP}$ and $\mathrm{HBP})$.

14. Comparison between groups in the correlation between changes from baseline to 12th, 24th, 36th and 48th month in albuminuria and changes from baseline to 12th, 24th, 36th and 48th month of different measures of $\mathrm{BP}(\mathrm{OBP}, \mathrm{ABP}$ and $\mathrm{HBP})$.

the central reading sites. The occurrence of $\mathrm{CV}$ events (stroke, myocardial infarction, all major CV events, CV death, all cause death) and renal outcomes reported by each centre and supported by clinical source documents (ie, diagnostic tests, medical reports) will be validated by an adjudicating committee of experts blinded to assigned management strategy, BP values and organ damage measurements.

\section{Data analysis plan}

Descriptive analysis

Continuous data will be summarised by means of mean value, median, minimum, maximum, SE, SD and number of observations. Moreover, a 95\% CI for the mean value will be provided. Categorical data will be summarised by means of absolute and relative frequencies. All these analyses will be performed per management group as well as for the study population as a whole. Unpaired t-test (or Mann-Whitney U test in case of non-normal distribution) for continuous variables or $\chi^{2}$ test for categorical ones in order to evaluate significant between-group differences at baseline will be applied. The incidence of adverse events will be tabulated by treatment group.

\section{Statistical methods for coprimary endpoint analysis}

We will consider two coprimary endpoints: change from baseline to 12th month in LVMI and in albumin:creatinine ratio. Each endpoint will be analysed by means of the analysis of covariance, adjusting the group effect for the baseline value of LVMI (or albumin:creatinine ratio) and for baseline covariates resulting significantly different between groups. Moreover, to have a valid inference, as suggested by several authors, we will adjust the model for all variables used in the dynamic allocation scheme. ${ }^{14}$ The assumption of parallelism will be tested by introducing one or more crossproduct terms between groups and continuous covariates to the model. 


\section{Statistical methods for secondary endpoint analysis}

For all secondary endpoints, except for endpoint 8 , we will consider a repeated measurement approach based on mixed models which contain both fixed effects (eg, BP measurement technique) and random effects (eg, patient). These models are likelihood-based approaches in the presence of ignorable missing data (ie, missing at random) and are a proper way to accommodate information on a patient with postrandomisation outcomes, even when such a patient's profile is incomplete. ${ }^{24}$ In particular, for endpoints 1, 3, 4, 5 and 7, a repeated-measures model for dichotomous endpoints will be applied, while for endpoints 2, 6, 9 and 10, we will consider a model for continuous outcome. In both cases, the main purpose will be to determine whether the within-person changes over time vary across levels of one or more betweenperson factors (eg, BP measurement technique and for the same covariates included in the model for coprimary endpoints). ${ }^{14}$ For endpoint 8 , cumulative proportion surviving curves according Kaplan-Meier will be obtained for each group and compared with the method of log-rank test. In order to explore the group effect on the timetoevent of composite outcome adjusting for baseline covariates, the Cox regression model will be fitted. Though the Cox model is non-parametric to the extent that no assumptions are made about the form of the baseline hazards, two important issues of non-informative censoring and proportional hazards will need to be verified. To satisfy the first assumption, the design of the underlying study must ensure that the mechanisms giving rise to censoring of individual subjects are not related to the probability of an event occurring. Care will be taken that continuation of follow-up does not depend on a participants medical condition. To satisfy the second assumption, the $\chi^{2}$ tests of proportional hazards assumption and the log-cumulative hazard plots will be made. If the assumption of proportionality is deemed reasonable, the assumptions of linearity and additivity, which are implicit in the linear predictor formula, will be considered. Although the composite endpoint could be misleading, we will not proceed to analyse each individual event considering the other events as competing causes of failure because also competing risk analysis can be misleading. Randomised controlled trials are frequently not powered to detect an effect of the intervention on individual components and competing risks analysis might have a low chance of detecting a true effect. ${ }^{25}$

\section{Statistical methods for tertiary endpoint analysis}

For endpoints 1-3, we will apply mixed models proposed for secondary endpoints. For endpoints 4-12, we will apply the $\chi^{2}$ test for dichotomous endpoints, the t-test (or median test whenever necessary) for continuous endpoints and, specifically for variation coefficients, the large sample $\mathrm{Z}$ test as suggested by Bhoj et al. ${ }^{26}$ For endpoints 13 and 14, the differences between the Pearson correlation coefficients of two groups will be calculated and reported with their 95\% CI using the approach proposed by Zou. ${ }^{27}$ The handling of missing data will be based on a multiple imputation approach. ${ }^{24}$ All statistical tests will be interpreted at the $5 \%$ significance level considering two-sided test, unless specified otherwise. All statistical analyses will be performed by the Statistical Centre of Istituto Auxologico Italiano, Milan. Statistical packages will be used for the analyses, chosen among Stata (STATA data analysis and statistical software, Texas, USA), R (The $\mathrm{R}$ project for statistical computing, free software) and SAS (SAS Institute, Cary, North Carolina, USA).

\section{Ethical considerations}

MASTER study protocol has initially received approval by the ethics committee of the Istituto Auxologico Italiano. Thus, the ethical conduct of this study will be under the control of an independent Data Safety Monitoring Board, stemming out of the Istituto Auxologico Italiano Ethical Review Board. This is an investigator-generated study and as such will be performed in full independence of the study sponsor from any other funding body. The procedures set out in this protocol, pertaining to the conduct, evaluation and documentation, are designed to ensure that the sponsor and investigator abide to Good Clinical Practice guidelines and follow the guiding principles detailed in the Declaration of Helsinki.

\section{Author affiliations}

${ }^{1}$ Department of Medicine and Surgery, University of Milan-Bicocca, Milan, Italy ${ }^{2}$ Istituto Auxologico Italiano, IRCCS, Department of Cardiovascular Neural and Metabolic Sciences, San Luca Hospital, Milan, Italy

${ }^{3}$ Centro Interuniversitario di Fisiologia Clinica e Ipertensione, University of Milan, Milan, Italy

${ }^{4}$ Department of Medicine, Azienda Spedali Civili di Brescia, Brescia, Italy ${ }^{5}$ Department of Clinical and Experimental Sciences, University of Brescia, Brescia, Italy

${ }^{6}$ Department of Medicine, University of Chicago, Chicago, USA

${ }^{7} \mathrm{ASH}$ Comprehensive Hypertension Center, Section of Endocrinology, Diabetes and metabolism, Chicago, USA

${ }^{8}$ Department of Cardiology, Università di Firenze, Florence, Italy

${ }^{9}$ Department of Hypertension and Diabetology, Medical University of Gdansk, Gdansk, Poland

${ }^{10}$ Department of Internal Medicine, Hospital Mutua Terrassa, University of Barcelona, Barcelona, Spain

${ }^{11}$ Department of Cardiovascular, Nutrition and Aging, Hospital Clinic of Barcelona, University of Barcelona, Insitut d'investigacions Biomèdiques August Pi I Sunyer (IDIBAPS), Barcelona, Spain

${ }^{12}$ Department of Cardiology, Emergency Clinical Hospital of Bucharest, Bucharest, Romania

${ }^{13}$ Department of Cardiology, Peking University First Hospital, Beijing, China

${ }^{14}$ Department of Nephrology, Hypertension, Dialysis and Transplantation, University of Zagreb School of Medicine, University Hospital Center Zagreb, Zagreb, Croatia

${ }^{15}$ Department of Clinical Sciences, Karolinska Institute, Stockholm, Sweden

${ }^{16}$ Division of Cardiovascular Medicine, Danderyd Hospital, Stockholm, Sweden

${ }^{17}$ Hypertension Department, Almazov Federeal North-Werst Medical Research Centre, St.Petersburg, Russian Federation

${ }^{18}$ Departments of Pharmacology, European Georges Pompidou Hospital, Assistance Publique Hôpitaux de Paris, Inserm UMR 970 and University Paris Descartes, Paris, France

${ }^{19}$ The Center of Hypertension of the Peoples Hospital, Urumqi, China

${ }^{20}$ Department of Cardiology, Dharma Vira Heart Center, Sir Ganga Ram Hospital, New Delhi, India

${ }^{21}$ Nuffield Department of Primary Care Health Sciences, University of Oxford, Oxford, UK 
${ }^{22}$ Department of Medicina Sperimentale e Clinica, University of Florence, Florence, Italy

${ }^{23}$ Fundacion Venezolana de Hipertensión Arterial, Instituto de investigaciones de Enfermedades Cardiovasculares de LUZ, Maracaibo, Venezuela, Bolivarian Republic of

${ }^{24}$ Clinical Research Unit, Italian Institute of Telemedicine, Solbiate Arno, Italy

${ }^{25}$ Dipartimento di Medicina DIMED, University of Padova, Padua, Italy

${ }^{26} \mathrm{JB}$ Lab and Clinic, Seoul, Korea (the Republic of)

${ }^{27}$ Department of Internal Medicine, Division of Cardiology, Medical University of Graz, Graz, Austria

${ }^{28}$ Department of Cardiology, County Clinical Hospital, University of Medicine and

Pharmacy Tirgu Mures, Tirgu Mures, Romania

${ }^{29}$ Department of Medicine, University of Perugia, Perugia, Italy

${ }^{30}$ Hypertension Clinic, Unit of Internal Medicine, "S.Maria" Hospital, Terni, Italy

${ }^{31}$ University of Valencia and INCLIVA Research Institute, Valencia, Spain

${ }^{32}$ Hypertension Clinic, Hospital Clinico de Valencia, Valencia, Spain

${ }^{33}$ Department of Cardiology, Hospital Español de Mendoza, Mendoza, Argentina

${ }^{34}$ Cardiovascular Center, Dongguk University Ilsan Hospital, Goyang-si/Gyeonggi-do, Korea (the Republic of)

${ }^{35}$ Department of Hypertension, Hospital de Sagunto and University CEU Cardenal Herrera, Ciencias de la Salud, Valencia, Spain

${ }^{36}$ Metabolic Unit, Hypertension section, Favaloro Foundation, Buenos Aires, Argentina

${ }^{37}$ Department of Nephrology and Hypertension, University Hospital Erlangen,

Erlangen, Germany

${ }^{38}$ Department of Statistics and Quantitative methods, University of Milan-Bicocca, Milan, Italy

${ }^{39}$ School of Medicine, Hypertension Center STRIDE-7, National and Kapodistrian University of Athens, Athens, Greece

${ }^{40}$ Third Department of Medicine, Sotiria Hospital, Athens, Greece

${ }^{41}$ Excellence Centre for Hypertension, Department of Endocrinology, School of Medicine, University of Belgrade, Belgrade, Serbia

${ }^{42}$ First Cardiology Clinic, Medical School, National and Kapodistrian University of Athens, Hippokration Hospital, Athens, Greece

${ }^{43}$ School of Medicine and Surgery, Center of Biostatistics for Clinical Epidemiology, University of Milan-Bicocca, Milan, Italy

${ }^{44}$ Department of Medical Sciences, Internal Medicine and Hypertension Division, Azienda 0spedaliero-Universitaria Città della Salute e della Scienza di Torino, Turin, Italy

${ }^{45}$ Department of Internal Medicine, Hypertension Section, Hospital Italiano de Buenos Aires, Buenos Aires, Argentina

${ }^{46}$ School of Medicince, The Shanghai Institute of Hypertension, Ruijin Hospital; Shanghai Jiaotong University, Shanghai, China

${ }^{47}$ Department of Hypertension, Centre For Epidemiological Studies And Clinical Trials, Shanghai Key Laboratory Of Hypertension, Shanghai, China

${ }^{48} \mathrm{Fu}$ Wai Hospital, Chinese Academy of Medical Sciences and Peking Union Medical Colleges, Beijing, China

Acknowledgements The names of the remaining investigators of the MASTER Study are listed in the supplementary file 1.

Contributors GP, RS, GS, RM, JR, SO, GLB, GM and AZ: contributed to the conception and design of the study. GP, RS, GM and AZ: drafted and wrote the protocol in accordance to the coauthors' contributions. TF and PW: the study managers in charge of database management and study monitoring. All authors contributed to the writing and the reviewing of this article, and approved the final draft of the protocol.

Funding This work is being supported only by unrestricted and non-conditioning grants by Pfizer (Pfizer, New York, New York, USA) through an InvestigatorInitiated Research Agreement (duly signed on 24/11/2015); Daiichi Sankyo (Daiichi Sankyo Co., Daiichi Sankyo Building A/B 3-5-1, Nihonbashi-honcho, Chuo-ku, Tokyo 103-8426, Japan) through an Unrestricted Research Grant Agreement (duly signed on 11/02/2016); Omron (Omron Healthcare Europe B.V. , Scorpius 33, 2132 LR Hoofddorp, Netherlands) through an Unrestricted Research Grant Agreement (duly signed on 18/11/2016); and Menarini (Menarini International Operations Luxembourg) through an Unrestricted Research Grant Agreement (duly signed on 27/06/2017). In order to standardise ABPMand OBPM data collection and analysis as much as possible, 24h ABPMin MASTER Study will be preferentially performed with A\&DTM2430 device and OBP levels will be measured with A\&DUA-651BLE device provided by the Coordinating center, thanks to a non-conditioning support given by the A\&D Company (A\&D Engineering, San Jose, California, USA).

Competing interests GP: honoraria as lecturer for Pfizer, Daiichi Sankyo, Menarini, Omron Healthcare. EA-R: honoraria and/or support from Menarini, Servier, Recordati,Guidotti,Malesci, Ferrer, DOC gen, Bruno farm. GLB: principal investigator (FIDElio)-Bayer, Steering committee (CREDENCE(Janssen), SONAR (AbbVie)Consultant for Merck, Relypsa, Vascular Dynamics, Elceyx, Bayer, Janssen, AbbVie. FC: collaboration with Smart Solutions Technologies S.A.; AMICUS; Boston Scientific International S.A . ADS: honoraria as lecturer for Abbott, Daiichi-Sankyo, Lacer, Menarini, and Pfizer. MD: Honoria from Recordati and Servier. TK: Research grants Karolinska Institutet from Amgen, Medtronic, Pfizer, and Record, all outside the presented work. SL: Honoraria for lecturing from Axelife, Daichi-Sankyo, FukudaDenshi, Menarini, Novartis, Omron, Servier and Recordati. GM: honoraria aslecturer Actavis, Amgen, Boehringer Ingelheim, CVRx, Daiichi Sankyo, Ferrer, Medtronic, Menarini, Merck, Novartis, Recordati, Sanofi,Servier. RJM: has received BP Monitors forresearch use from Omron. JR: hasbeen paid as lecturer by Daiichi Sankyo, Menarini, Boehringerlngelheim, MSD. MYR: Lecture honoraria from Pfizer,LG Life Sciences, Bayer Korea, Hanmi Pharm. Co., Yuhan Co., Boryung Pharmaceutical Co., Research grant fromBoryung Pharmaceutical Co. and Dong-A Pharmaceutical Co., CJ HealthCare Co. GS: Conductedvalidation studies for various manufacturers; advised manufacturerson device development. JGW: lecture and consulting fees from Bayer, Daiichi-Sankyo, Novartis,0mron, Pfizer, Sanofi, and Servier. AZ: Honoraria from Menarini International.

Patient consent Not required.

Ethics approval MASTER study protocol has initially received approval by the Ethical Review Board of the Istituto Auxologico Italiano. All centresin this study will have to obtain approval from Ethics Review Committees and/or Institution also in their country in order to participate, and the study should be carried out in line with applicable local law(s) and regulation(s). The need of study insurance will be determined for each participating centre according to national and local laws.

Provenance and peer review Not commissioned; externally peer reviewed.

Open access This is an open access article distributed in accordance with the Creative Commons Attribution Non Commercial (CC BY-NC 4.0) license, which permits others to distribute, remix, adapt, build upon this work non-commercially, and license their derivative works on different terms, provided the original work is properly cited, appropriate credit is given, any changes made indicated, and the use is non-commercial. See: http://creativecommons.org/licenses/by-nc/4.0/.

\section{REFERENCES}

1. Franklin SS, Thijs L, Hansen TW, et al. Significance of white-coat hypertension in older persons with isolated systolic hypertension: a meta-analysis using the International Database on Ambulatory Blood Pressure Monitoring in Relation to Cardiovascular Outcomes population. Hypertension 2012;59:564-71.

2. Banegas JR, Ruilope LM, de la Sierra A, et al. High prevalence of masked uncontrolled hypertension in people with treated hypertension. Eur Heart J 2014;35:3304-12.

3. Franklin SS, Thijs L, Li Y, et al. Masked hypertension in diabetes mellitus: treatment implications for clinical practice. Hypertension 2013;61:964-71.

4. Pierdomenico SD, Lapenna D, Bucci A, et al. Cardiovascular outcome in treated hypertensive patients with responder, masked, false resistant, and true resistant hypertension. Am J Hypertens 2005;18:1422-8.

5. Mancia G, Fagard R, Narkiewicz K, et al. 2013 ESH/ESC guidelines for the management of arterial hypertension: the Task Force for the Management of Arterial Hypertension of the European Society of Hypertension (ESH) and of the European Society of Cardiology (ESC). Eur Heart J 2013;34:2159-219.

6. Parati G, Stergiou G, O'Brien E, et al. European Society of Hypertension practice guidelines for ambulatory blood pressure monitoring. J Hypertens 2014;32:1359-66.

7. O'Brien E, Parati G, Stergiou G, et al. European Society of Hypertension position paper on ambulatory blood pressure monitoring. J Hypertens 2013;31:1731-68.

8. Parati G, Stergiou GS, Asmar R, et al. European society of hypertension guidelines for blood pressure monitoring at home: a summary report of the second international consensus conference on home blood pressure monitoring. J Hypertens 2008;26:1505-26.

9. Weber MA, Schiffrin EL, White WB, et al. Clinical practice guidelines for the management of hypertension in the community: a statement by the American Society of hypertension and the international society of hypertension. J Clin Hypertens 2014;16:14-26. 
10. Shimamoto K, Ando K, Fujita T, et al. The Japanese society of hypertension guidelines for the management of hypertension (JSH 2014). Hypertens Res 2014;37:253-390.

11. Piper MA, Evans CV, Burda BU, et al. Diagnostic and predictive accuracy of blood pressure screening methods with consideration of rescreening intervals: a systematic review for the u.s. preventive services task force. Ann Intern Med 2015;162:192-204.

12. NICE. Hypertension: the clinical management of primary hypertension in adults: update of clinical guidelines 18 and 3. 2011;127 wwwniceorguk/guidance/CG127.

13. Xiao L, Yank V, Ma J. Algorithm for balancing both continuous and categorical covariates in randomized controlled trials. Comput Methods Programs Biomed 2012;108:1185-90.

14. Hu F, Hu Y, Ma Z, et al. Adaptive randomization for balancing over covariates. Wiley Interdiscip Rev Comput Stat 2014;6:288-303.

15. Marwick TH, Gillebert TC, Aurigemma G, et al. Recommendations on the use of echocardiography in adult hypertension: a report from the European Association of Cardiovascular Imaging (EACVI) and the American Society of Echocardiography (ASE). J Am Soc Echocardiogr 2015;28:727-54.

16. Chirinos JA, Segers P, De Buyzere ML, et al. Left ventricular mass: allometric scaling, normative values, effect of obesity, and prognostic performance. Hypertension 2010;56:91-8.

17. Lauer MS, Anderson KM, Kannel WB, et al. The impact of obesity on left ventricular mass and geometry. the framingham heart study. JAMA 1991;266:231-6.

18. Lang RM, Badano LP, Mor-Avi V, et al. Recommendations for cardiac chamber quantification by echocardiography in adults: an update from the American Society of Echocardiography and the European
Association of Cardiovascular Imaging. J Am Soc Echocardiogr 2015;28:1-39.

19. Nagueh SF, Appleton CP, Gillebert TC, et al. Recommendations for the evaluation of left ventricular diastolic function by echocardiography. J Am Soc Echocardiogr 2009;22:107-33.

20. Nagueh SF, Smiseth OA, Appleton CP, et a/Recommendations for the evaluation of left ventricular diastolic function by echocardiography: an update from the american society of echocardiography and the european association of cardiovascular imaging. J Am Soc Echocardiogr 2016;29:277-314.

21. Dahlof B, Zanchetti A, Diez J, et al. Effects of losartan and atenolol on left ventricular mass and neurohormonal profile in patients with essential hypertension and left ventricular hypertrophy. J Hypertens 2002;20:1855-64.

22. Cuspidi C, Muiesan ML, Valagussa L, et al. Comparative effects of candesartan and enalapril on left ventricular hypertrophy in patients with essential hypertension: the candesartan assessment in the treatment of cardiac hypertrophy (CATCH) study. J Hypertens 2002;20:2293-300

23. Galli F, Mariani L. Two-stage re-estimation adaptive design: a simulation study. Epidemiology, Biostatistics and Public Health 2013;11.

24. Molenberghs $\mathrm{G}$, Thijs $\mathrm{H}$, Jansen I, et al. Analyzing incomplete longitudinal clinical trial data. Biostatistics 2004;5:445-64.

25. Wolbers M, Koller MT, Stel VS, et al. Competing risks analyses: objectives and approaches. Eur Heart J 2014;35:2936-41.

26. Bhoj DS, Ahsanullah M. Testing equality of coefficients of variation of two populations. Biometrical Journal 1993;35:355-9.

27. Zou GY. Toward using confidence intervals to compare correlations. Psychol Methods 2007;12:399-413. 